Nonlinear Processes in Geophysics (2005) 12: 13-24

SRef-ID: $1607-7946 / n p g / 2005-12-13$

European Geosciences Union

(C) 2005 Author(s). This work is licensed

under a Creative Commons License.

\title{
Nonlinear dynamics of turbulent waves in fluids and plasmas
}

\author{
K. $\mathrm{He}^{1}$ and A. C.-L. Chian ${ }^{2}$ \\ ${ }^{1}$ Inst. of Low Ener. Nucl. Phys., Beijing Normal Univ., Beijing, 100875 China \\ ${ }^{2}$ National Institute for Space Research (INPE), P.O. Box 515,12227-010, São José dos Campus-SP, Brazil \\ Received: 14 September 2004 - Revised: 23 November - Accepted: 15 December 2004 - Published: 4 January 2005 \\ Part of Special Issue "Nonlinear processes in solar-terrestrial physics and dynamics of Earth-Ocean-System"
}

\begin{abstract}
In a model drift wave system that is interesting both in fluids and plasmas, we find that an embedded moving saddle point plays an important role at the onset of turbulence. Here the saddle point is actually a saddle steady wave, in its moving frame the wave system can be transformed into a set of coupled oscillators whose motion is affected by the saddle steady wave as if it is a potential. It is found that a collision with the saddle point triggers a crisis, following the collision another dynamic event occurs which involves a transition in the phase state of the master oscillator. Only after the latter event the spatial regularity is destroyed. The phase dynamics before and after the transition is further investigated. It is found that in a spatially coherent state before the transition the oscillators reach a functional phase synchronization collectively with or without phase slips, after the transition in the turbulent state an on-off imperfect synchronization is established among the oscillators with long wavelengths. When the synchronization is on, their amplitudes grow up simultaneously, giving rise to a burst in the total wave energy. A power law behavior is observed in the correlation function between phases of the oscillators. Potential application of our results in prediction of energy bursts in turbulence is discussed.
\end{abstract}

\section{Introduction}

Turbulence can be observed in systems of very different in scales and properties, which is an important topic in a variety of disciplines (Frisch, 1995). In the sun and other stars, flares and electromagnetic emissions are observed, it has been suggested that, e.g. solar flares are caused by nonlinear dynamics of solar plasma turbulence (Boffetta et al., 1999). In our environment, e.g. in space, oceans and atmosphere, turbulence is often encountered, which impact on human lives for thousands of years. In biological systems turbulence may play an

Correspondence to: $\mathrm{K} . \mathrm{He}$

(kfhe@bnu.edu.cn) active role, e.g. brain dynamics is turbulent when it is awake (Kandel et al., 2000).

In contrast to wide awareness of turbulence, people knew little about its mechanism until recently when great progress has been made in the investigation of nonlinear dynamics. A great deal of experimental evidence has been found that strengthens the confidence that turbulence can be understood on the basis of nonlinear dynamics. Some scenarios to chaos, e.g. period-doubling, Ruelle-Takens route as well as intermittency, have found supports from experimental and theoretical investigations (Lichtenberg and Lieberman, 1983; Infeld and Rowlands, 1990; Eckmann, 1981; Swinney, 1983; Biskamp and He, 1985; Klinger et al., 1997). This progress encourages scientists to further study turbulence in spatially extended systems. In this area abundant phenomena, such as various space-time patterns, solitons, weak and strong turbulence are not yet fully understood and worthwhile to be explored (Cross and Hohenberg, 1993; Bishop et al., 1983; Chaté and Manneville, 1987; He and Salat, 1989; Chian et al., 2002). In this paper we will present some results of our investigation on dynamics of nonlinear waves, the attention will be focused on the questions as what causes a spatially regular wave to transit to a spatiotemporal chaotic wave or a turbulence. In our discussion effect of a saddle point and phase dynamics will be emphasized.

What is a saddle point in a spatially extended system? How did we realize that it may play an important role at the onset of turbulence? Our conjecture on the role of saddle point in turbulence arises from a very interesting phenomenon in some experimental and numerical observations: a link seems to exist between chaotic or turbulent waves and hystereses (Nakatsuka et al., 1983; Sun et al., 1995; Chern and I, 1991; Foss et al., 1996; Kim et al., 1997; He and Salat, 1989). We have studied several nonlinear wave systems where the steady wave energies constitute hystereses (He and Salat, 1989, 1988; Zhou and He, 1998), of which in a driven/damped drift-wave system (see Eq. 1) we found very turbulent wave solutions, in particular the regimes where turbulent solutions are observed seem to have a relation with the 
regimes where hystereses exist (He and Salat, 1989). Another significant phenomenon is: when its wave energy is in the negative tangential branch of an S-shaped hysteresis a steady wave solution is unstable due to a saddle-node instability. Such a saddle steady wave can not be realized, it is a virtual wave embedded in certain realized waves, e.g. in some stable solitary waves, or in periodic, chaotic and turbulent waves. These phenomena lead to our conjecture that an embedded saddle steady wave may play an important role at the onset of turbulence.

In general, a steady wave solution is like a solitary wave, which moves in a constant group velocity with an invariable shape. If observed in the reference frame moving with this group velocity, a steady wave solution is a fixed point in the Fourier space. With variation of applied parameters such a fixed point may lose its stability, just like what happens in time-dependent systems. If the instability is of saddle type, this steady wave solution is a saddle point. Therefore, in a reference frame moving with its group velocity, a saddle steady wave solution is an embedded saddle point, in another word, in the lab frame it is a moving saddle point embedded in the realized wave.

Indeed we have found convincing evidence in the driven/damped drift-waves that such an embedded saddle point plays a critical role at the onset of turbulence. We find that a collision with the saddle point triggers a crisis. However, this collision does not directly destroy the spatial coherence. Then a further question is: how the spatial coherence is destroyed? To answer this question the motion of different spatial scales has to be studied. To this end we transform the wave system to a set of coupled oscillators moving in a potential of the saddle steady wave solution, and find that subsequently to the collision there occurs another critical event during which the phase of the master oscillator experiences a trapped-free transition. It is this event that plays a key role in the destruction of spatial coherence. These results will be given in Sect. 2. In the following sections we investigate the dynamics of the oscillators, especially of their phases. We find that, in spatially regular wave before the transition perfect functional relations are formed between the mode phases (ref. Sect. 3), in a turbulent wave after the transition the oscillators show much stronger tendency to establish a phase synchronization (PS), however, due to the embedded saddle point they are not easy to reach a perfect PS, instead, an onoff imperfect PS is established among the oscillators (ref. Sect. 4). At "on" stages of the synchronization amplitudes of the oscillators of different scales grow up almost simultaneously, inducing bursts in the total wave energy. This result presents an explanation for the dynamic cause of energy bursts in the turbulent motion. Finally Sect. 5 is a conclusion and discussion.

\section{Crisis-induced transition to spatiotemporal chaos}

In He (1998) we found a crisis-induced transition from a spatially regular to spatiotemporally chaotic wave in the follow- ing model equation,

$\frac{\partial \phi}{\partial t}+a \frac{\partial^{3} \phi}{\partial t \partial x^{2}}+c \frac{\partial \phi}{\partial x}+f \phi \frac{\partial \phi}{\partial x}=-\gamma \phi-\varepsilon \sin (x-\Omega t) .(1)$

Without driving $\varepsilon$ and damping $\gamma$ the system describes shallow water wave in fluids (Dodd et al., 1982; Benjamin et al., 1972) and drift wave in magnetized plasmas (Horton, 1990), so Eq. (1) has practical applications.

A steady wave solution of Eq. (1) has a form of $\phi_{0}(x-\Omega t)$, which is a fixed point in the reference frame $\xi=x-\Omega t, \tau=t$. Here $\phi_{0}(\xi)$ obeys a steady equation,

$\frac{\partial \phi_{0}}{\partial \tau}=0$

By expanding $\phi_{0}(\xi)=\sum_{k} A_{k} \cos \left(k \xi+\theta_{k}\right)$ (here and in the following $k=1,2, \cdots, N \rightarrow \infty)$ and with appropriate guessed values one can work out its mode amplitudes and phases, $\left\{A_{k}, \theta_{k}\right\}$, then $\phi_{0}(\xi)$ is obtained. Its wave energy, $E_{0} \equiv E\left(\phi=\phi_{0}\right)$, is a constant, here

$E(t)=\frac{1}{2 \pi} \int_{0}^{2 \pi} \frac{1}{2}\left[\phi^{2}(t)-a \phi_{x}^{2}(t)\right] d x$.

It has been found that for given $\Omega$ in certain regimes steady wave energy $E_{0}$ as a function of $\varepsilon$ forms an S-hysteresis. Stability analysis shows that when its energy locates in the negative tangential branch of a hysteresis a steady wave solution $\phi_{0}$ is unstable due to a saddle-node instability, such a saddle steady wave is denoted as $\phi_{0}^{*}(\xi)$ in the following, in the $(\xi, \tau)$ frame it is a saddle point in the Fourier space. In the following we will see that this saddle point plays a critical role in the transition to turbulence. On the other hand, if its wave energy locates in the lower branch of a S-hysteresis a steady wave solution $\phi_{0}(x-\Omega t)$ can be stable, or it may lose the stability through a Hopf bifurcation (He , 2004).

To study the complicated dynamic behaviors we have used the pseudospectral method to solve Eq. (1) in periodic boundary condition, $\phi(x+2 \pi)=\phi(x)$. In the chosen parameter regimes $2^{6}$ to $2^{9}$ spatial grids are tested, the results are in agreement with each other. Among others, we found that for a given $\Omega$ in certain regime there exists a critical $\varepsilon=\varepsilon_{c}$, before which the wave is spatially regular although it can be erratic in time, but after which spatial regularity of the wave is destroyed, the wave looks very turbulent, in particular it bears some characteristic feature of fully-developed turbulence, e.g. a power law spatial spectrum. For $\Omega=0.65$ the critical transition point $\varepsilon_{c} \approx 0.20$. Figure 1 shows the typical space-time contour plot of (a) spatially regular wave where one can still see a wave structure and (b) spatiotemporally chaotic wave where the wave is broken.

For a given $\varepsilon>\varepsilon_{c}$ a sudden enlargement of the attractor can be seen in the transient state. It is along an unstable orbit of the saddle steady wave $\phi_{0}^{*}(x-\Omega t)$ that the realized trajectory is led to the spatiotemporal chaotic attractor (He, 1998), similar to what happens in a type of crisis in time-dependent systems (Ott, 1993). We also find that the transition is triggered by a "saddle pattern resonance", that is, a crisis occurs 
(a)
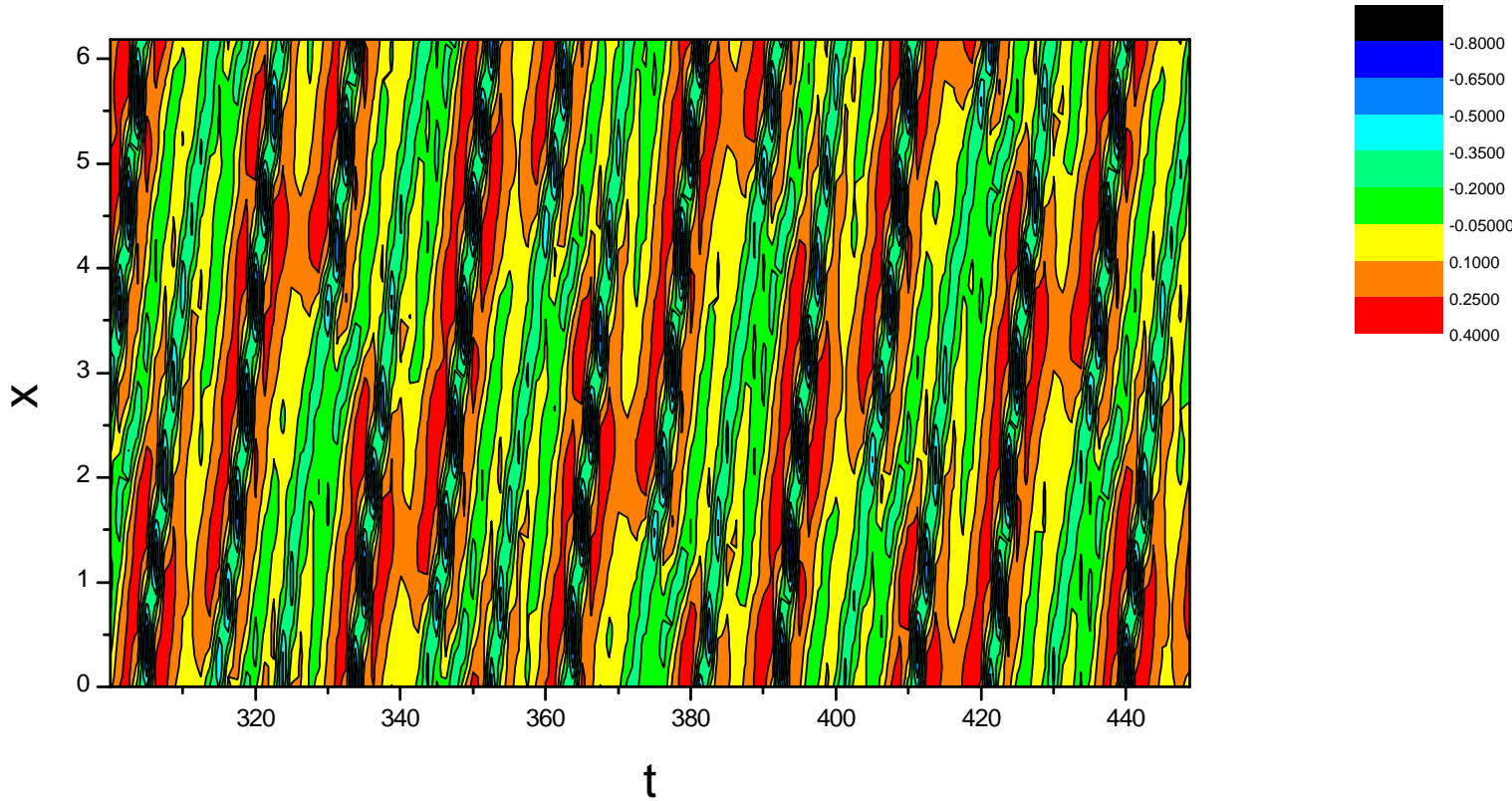

(b)
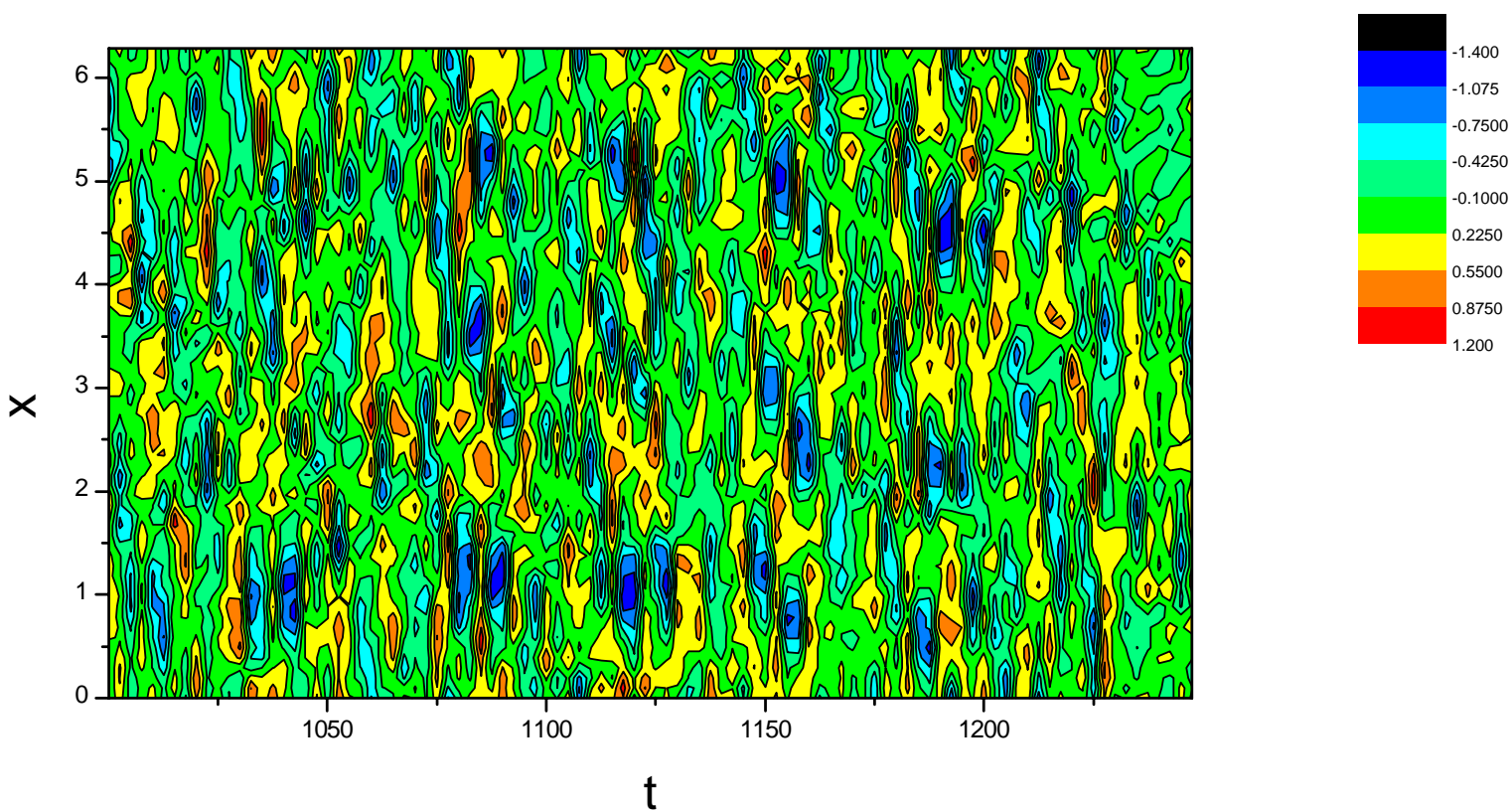

Fig. 1. Space-time contour plot of $\phi(x, t)$ for (a) spatially regular wave before the transition with $\varepsilon=0.19$, (b) spatiotemporally chaotic wave after the transition with $\varepsilon=0.22 ; \Omega=0.65$.

when the realized wave $\phi(x, t)$ evolves to about the same shape of saddle steady wave $\phi_{0}^{*}(x-\Omega t)(\mathrm{He}, 2000)$. Figure 2 shows a contour plot of $\Delta^{2}(x, t)$ for $\Omega=0.65, \varepsilon=0.22$, here $\Delta(x, t) \equiv\left|\phi(x, t)-\phi_{0}^{*}(x-\Omega t)\right|$ is the space-time difference between the realized wave and saddle steady wave. In this example when $t<\sim 30$ the realized wave $\phi(x, t)$ is spatially regular, when $t>\sim 50$ it transits to a state of extreme irregularity both in time and in space. In the plot one can see that just before the transition there appears a zone with almost white color, indicating that at this time interval the discrepancy between waveforms of $\phi(x)$ and $\phi_{0}^{*}(x)$ becomes very small. Indeed a 'pattern resonance' occurs at $t=t^{*} \approx 39$ as indicated by an arrow in the plot. Figure 3 depicts the waveforms of $\phi\left(x, t^{*}\right)$ and $\phi_{0}^{*}\left(x-\Omega t^{*}\right)$ at the critical time $t^{*}$, one can see that the former almost coincides with the latter. Normally $\phi_{0}^{*}(x-\Omega t)$ is virtual, because of its instability 

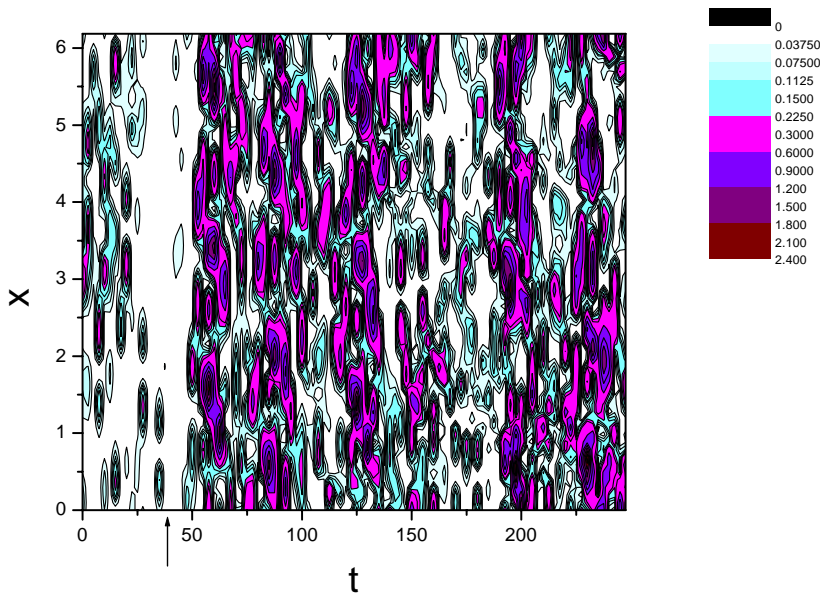

Fig. 2. Contour plot of space-time distance between the realized wave and the saddle steady wave, $\Delta^{2}(x, t) \equiv\left|\phi(x, t)-\phi_{0}^{*}(x-\Omega t)\right|^{2}$, $\Omega=0.65, \varepsilon=0.22$.

this solution exists only theoretically; however, just at $t \approx t^{*}$ the saddle steady wave $\phi_{0}^{*}\left(x-\Omega t^{*}\right)$ appears approximately in reality, in this case a disturbance to it would grow along its unstable orbit, a crisis then sets in which finally leads to the spatiotemporal chaotic attractor.

Since a saddle steady wave is a saddle point in the $(\xi, \tau)$ frame, we anticipate that a collision with the saddle point can be observed at onset of the crisis(Ott, 1993). With this consideration at first we project the orbit onto a Poincaré section, the one that was used in $\mathrm{He}$ (1998), unfortunately in this representation we failed to see such a collision, an apparent gap always shows up between the realized orbit and the saddle point at the onset of crisis. Later on, we realized the reason why the collision can not be manifested in a Poincaré section: although at the critical time $t^{*}$ the realized wave $\phi\left(x, t^{*}\right)$ evolves to a shape very similar to that of the virtual wave $\phi_{0}^{*}\left(x-\Omega t^{*}\right)$, the two waveforms are never exactly the same (see Fig. 3), in particular the discrepancy between their high $k$ modes can be apparent. In fact, a representation in which the collision can be observed should be in accordance with what happens at the onset of crisis, that is, in accordance with the phenomenon of "pattern resonance". With this in mind we choose a representation $\partial \phi(\xi=0, \tau) / \partial \xi$ vs. $\phi(\xi=0, \tau)$ in which such a collision can be fairly well demonstrated (He and Chian, 2004).

Instead of pseudospectral method applied to obtain Figs. 1, 2 and 3, for demonstrating the collision and other mechanisms we prefer to use a different approach. Since in $(\xi, \tau)$ frame a steady wave solution $\phi_{0}(\xi)$ is a fixed point, it allows us to set $\phi(\xi, \tau)=\phi_{0}(\xi)+\delta \phi(\xi, \tau)$, then from Eq. (1) the active part of the wave, $\delta \phi(\xi, \tau)$, is governed by the following equation,

$$
\begin{aligned}
& \frac{\partial}{\partial \tau}\left[1+a \frac{\partial^{2}}{\partial \xi^{2}}\right] \delta \phi-\Omega \frac{\partial}{\partial \xi}\left[1+a \frac{\partial^{2}}{\partial \xi^{2}}\right] \delta \phi+c \frac{\partial}{\partial \xi} \delta \phi \\
& +\gamma \delta \phi+f \frac{\partial}{\partial \xi}\left[\phi_{0}(\xi) \delta \phi\right]+f \delta \phi \frac{\partial}{\partial \xi} \delta \phi=0 .
\end{aligned}
$$

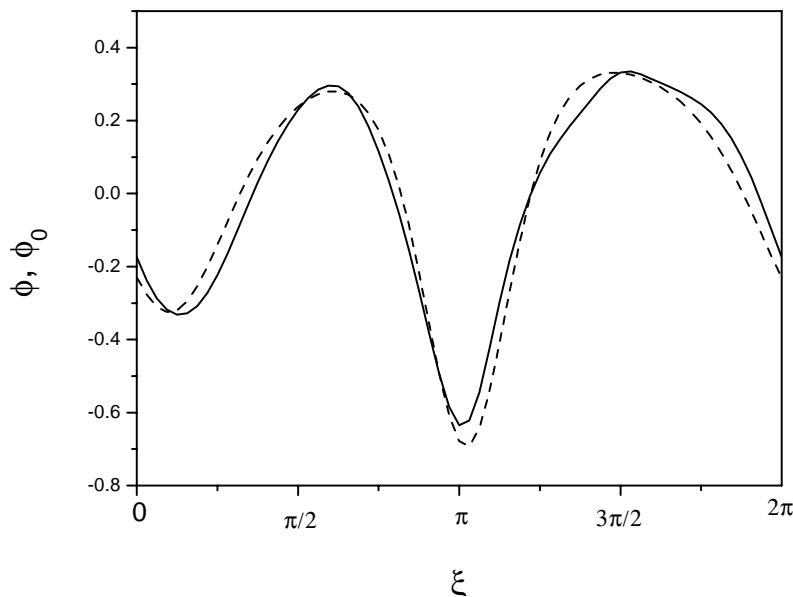

Fig. 3. Waveforms of the realized wave $\phi\left(x, t^{*}\right)$ (solid line) and the virtual saddle steady wave $\phi_{0}^{*}\left(x-\Omega t^{*}\right)$ (dashed line) at the critical time of 'pattern resonance', $t^{*} \approx 39.0$, the same case as in Fig. 2.

Substituting the expansion of $\phi_{0}(\xi)$ as well as $\delta \phi(\xi$, $\tau)=\sum_{k} b_{k}(\tau) \cos \left[k \xi+\alpha_{k}(\tau)\right]$ into Eq. (4) we obtain a set of ordinary differential equations for the modes $\left\{b_{k}(\tau), \alpha_{k}(\tau)\right\}$ of $\delta \phi(\xi, \tau)$ :

$$
\begin{aligned}
& \frac{d b_{k}}{d \tau}=-\frac{\gamma}{1-a k^{2}} b_{k}+\frac{f k}{2\left(1-a k^{2}\right)} \\
& \times\left\{\sum_{i+j=k}\left[A_{i} b_{j} \sin \left(\theta_{i}+\alpha_{j}-\alpha_{k}\right)+b_{i} b_{j} \sin \left(\alpha_{i}+\alpha_{j}-\alpha_{k}\right) / 2\right]\right. \\
& +\sum_{i-j=k}\left[A_{i} b_{j} \sin \left(\theta_{i}-\alpha_{j}-\alpha_{k}\right)+b_{i} b_{j} \sin \left(\alpha_{i}-\alpha_{j}-\alpha_{k}\right) / 2\right] \\
& \left.\sum_{j-i=k}\left[A_{i} b_{j} \sin \left(-\theta_{i}+\alpha_{j}-\alpha_{k}\right)+b_{i} b_{j} \sin \left(-\alpha_{i}+\alpha_{j}-\alpha_{k}\right) / 2\right]\right\}, \\
& \frac{d \alpha_{k}}{d \tau}=-k\left[\frac{c}{1-a k^{2}}-\Omega\right]-\frac{f k}{2\left(1-a k^{2}\right) b_{k}} \\
& \times\left\{\sum_{i+j=k}\left[A_{i} b_{j} \cos \left(\theta_{i}+\alpha_{j}-\alpha_{k}\right)+b_{i} b_{j} \cos \left(\alpha_{i}+\alpha_{j}-\alpha_{k}\right) / 2\right]\right. \\
& +\sum_{i-j=k}\left[A_{i} b_{j} \cos \left(\theta_{i}-\alpha_{j}-\alpha_{k}\right)+b_{i} b_{j} \cos \left(\alpha_{i}-\alpha_{j}-\alpha_{k}\right) / 2\right] \\
& \left.+\sum_{j-i=k}\left[A_{i} b_{j} \cos \left(-\theta_{i}+\alpha_{j}-\alpha_{k}\right)+b_{i} b_{j} \cos \left(-\alpha_{i}+\alpha_{j}-\alpha_{k}\right) / 2\right]\right\} .
\end{aligned}
$$

Owing to the last term in the left hand side of Eq. (4), modes $b_{k}(\tau)$ and $\alpha_{k}(\tau)$ of different wavenumber $k$ are coupled with each other; besides, their motion is also affected by the steady wave solution $\phi_{0}(\xi)$ as if the latter is a potential well. Therefore, with Eqs. (5) we have transformed the nonlinear wave Eq. (1) into a set of coupled oscillators $\left\{b_{k}(\tau), \alpha_{k}(\tau)\right\}$ in a potential $\phi_{0}(\xi)$. After the modes of $\phi_{0}(\xi)$, i.e. $\left\{A_{k}, \theta_{k}\right\}$, are obtained, temporal variations of $\left\{b_{k}(\tau), \alpha_{k}(\tau)\right\}$ and hence $\delta \phi(\xi, \tau)$ can be worked out. Since $\phi_{0}(\xi)$ is a solitary wavelike structure which has few discrete lines in its spectrum, the active part $\delta \phi(\xi, \tau)$ is actually responsible for the abundant wave patterns observed in this system. In the following we use a saddle steady wave $\phi_{0}^{*}(\xi)$ as the potential and calculate variations of the corresponding $\delta \phi(\xi, \tau)$. Since $\phi_{0}^{*}(\xi)$ 

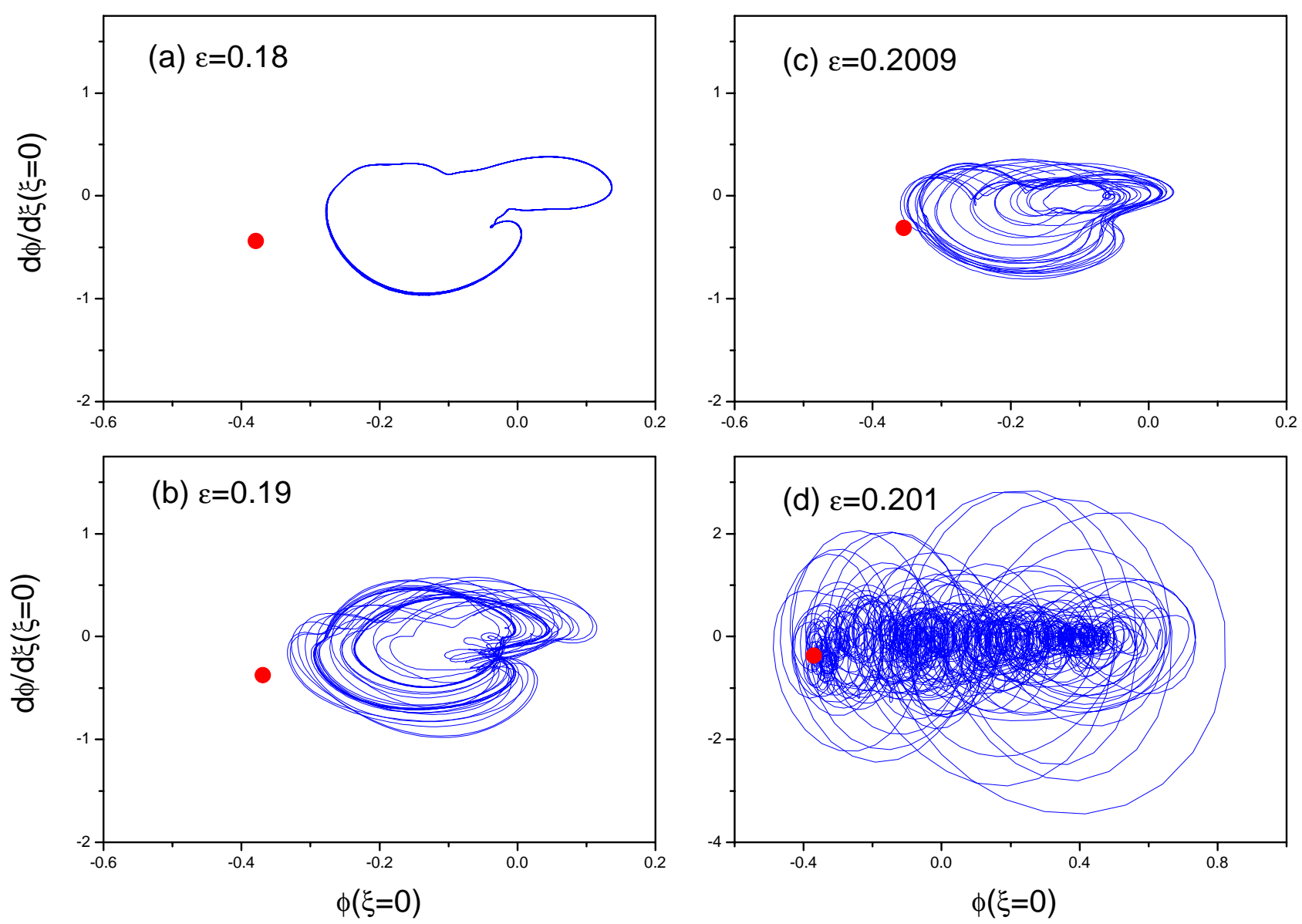

Fig. 4. Asymptotic attractor of $\phi(\xi, \tau)$ (solid line) and saddle point of $\phi_{0}^{*}(\xi)$ (bullet) in phase space $\partial \phi(0, \tau) / \partial \xi$ vs. $\phi(0, \tau)$ for $\Omega=0.65$ with (a) $\varepsilon=0.18$, (b) 0.19 , (c) 0.2009 before the crisis and (d) 0.201 after the crisis.

changes very little when crossing critical parameter $\varepsilon_{c}$, the dramatic changes in the dynamics when $\varepsilon>\varepsilon_{c}$ should be attributed to the active part $\delta \phi(\xi, \tau)$.

Now let us draw the orbit in phase plot $\partial \phi(0, \tau) / \partial \xi$ vs. $\phi(0, \tau)$, here

$\phi(\xi, \tau)=\sum_{k=1}^{N}\left\{A_{k} \cos \left(k \xi+\theta_{k}\right)+b_{k}(\tau) \cos \left(k \xi+\alpha_{k}(\tau)\right]\right\}$

$\partial \phi(\xi, \tau) / \partial \xi=-\sum_{k=1}^{N} k\left\{A_{k} \sin \left(k \xi+\theta_{k}\right)+b_{k}(\tau) \sin \left(k \xi+\alpha_{k}(\tau)\right]\right\}$,

and $\left\{A_{k}, \theta_{k}\right\}$ and $\left\{b_{k}(\tau), \alpha_{k}(\tau)\right\}$ are solved from the mode equations of Eq. (2) and Eqs. (5), respectively. The truncation number $N$ depends on the parameter regime. Specifically, since in the present regime the unstable orbit of saddle point is dominant by its $k=2$ component, $N$ should be sufficiently larger than 2 for demonstrating the crisis. We have tested 13-38 modes, the results are qualitatively the same, in particular in all the test runs collision with the saddle point can be well displayed.

For a given $\Omega$ one can see the collision when $\varepsilon$ is varied. Figure 4 shows the asymptotic attractors for $\Omega=0.65$ with Fig. $4 \mathrm{a}-\mathrm{c} \varepsilon=0.18,0.19,0.2009<\varepsilon_{c}$, Fig. $4 \mathrm{~d} \varepsilon=0.201>\varepsilon_{c}$, respectively. In the plot a saddle steady wave $\phi_{0}^{*}(\xi)$ is a (saddle) fixed point (denoted by a bullet) depending only on the applied parameters. In Fig. $4 \mathrm{a}-\mathrm{c}$ with $\varepsilon$ approaching to $\varepsilon_{c}$ the attractor and the saddle point are getting closer and closer. At the critical parameter $\varepsilon_{c}$ the attractor touches the saddle point. As soon as $\varepsilon$ crosses over $\varepsilon_{c}$ in Fig. $4 \mathrm{~d}$ the asymptotic attractor is greatly enlarged with the saddle point being overlapped by it. In this enlarged attractor $\partial \phi(0, \tau) / \partial \xi$ as a function of $\phi(0, \tau)$ changes with time violently, indicating that the spatial coherence has lost and the motion is very turbulent. This plot shows evidently that a collision with the saddle point is responsible for the dynamic transition from the spatially regular wave to spatiotemporal chaos in the parameter space.

For a fixed $\varepsilon>\varepsilon_{c}$ one can also see a transient collision with the saddle point. Figure 5 depicts phase plot $\partial \phi(0, \tau) / \partial \xi$ vs. $\phi(0, \tau)$ for a transient period of transiting from the spatially regular wave to spatiotemporal chaos for $\Omega=0.65, \varepsilon=0.22$. The initial distribution $\phi(\xi, \tau=0)$ is chosen adjacent to the saddle steady wave $\phi_{0}^{*}(\xi)$, to avoid confusion the first few steps of the orbit have been omitted. One can see that the orbit makes increasingly larger smooth circles and finally a collision with the saddle point occurs. After the collision the 


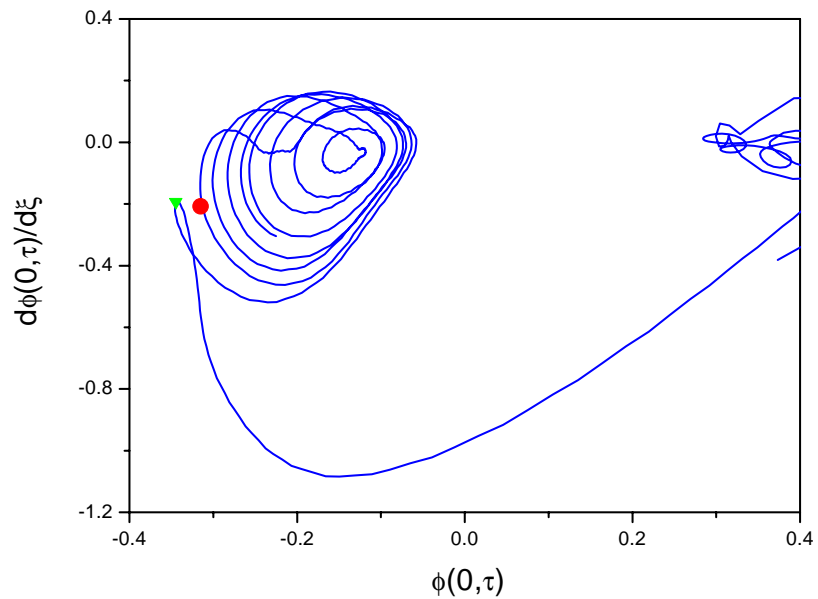

Fig. 5. Transient state of $\phi(\xi, \tau)$ (solid line) from the spatially regular wave to spatiotemporal chaos in phase space $\partial \phi(0, \tau) / \partial \xi$ vs. $\phi(0, \tau)$ for $\Omega=0.65, \varepsilon=0.22$, one can see a collision with the saddle point (bullet) and a turning point (triangle) of ejecting to the spatiotemporal chaotic attractor.

orbit continues to move in a smooth orbit for one circle, only when it gets nearer to the saddle point again, the orbit suddenly changes its orientation and turns to the spatiotemporal chaotic attractor.

In this representation the collision can be seen clearly, however, it does not mean that at the collision the orbit reaches precisely the position where the saddle point is. At the moment of collision the waveform of $\phi(\xi, \tau)$ almost coincides with that of $\phi_{0}^{*}(\xi)$ but the former is not exactly the same as the latter, just like what happens in Fig. 3. That is, transition to turbulence is induced by an approximate collision instead of an exact one. This result seems reasonable, for otherwise an infinitely small disturbance could halt the transition, that should not be the case in practical situations.

A surprising phenomenon in Fig. 5 is that the collision does not directly destroy the spatial coherence, in all the test runs one can see a remarkable turning point just about one circle after the collision as marked in Fig. 5 by a triangle, at which the orbit seems to be dragged by an unknown force to deviate from the smooth spatially regular attractor and turn to the spatiotemporal chaotic one, indicating that there must be an important event taking place here.

To understand what happens at the turning point we invoke Eqs. (5) again, but now we need the knowledge on the modes of $\delta \phi(\xi, \tau)$. Our investigation shows that the master oscillator, i.e. of $k=1$, plays a critical role in this respect. For the same case of Fig. 5, we plot $b_{1}(\tau)$ vs. $\alpha_{1}(\tau)$ in Fig. 6, in which the triangle corresponds exactly to the same critical moment at the turning point in Fig. 5. One can see that corresponding to the cyclic motion in Fig. 5, in Fig. $6 b_{1}(\tau)$ vs. $\alpha_{1}(\tau)$ makes vibrations like a nonlinear pendulum with $\alpha_{1}(\tau)$ confined in a range much less than $2 \pi$. Right at the critical moment of the turning point in Fig. 5, the orbit $b_{1}(\tau)$ vs. $\alpha_{1}(\tau)$ moves to the top of a hump in Fig. 6, after which $\alpha_{1}(\tau)$ no longer simply vibrates, instead it may cross over

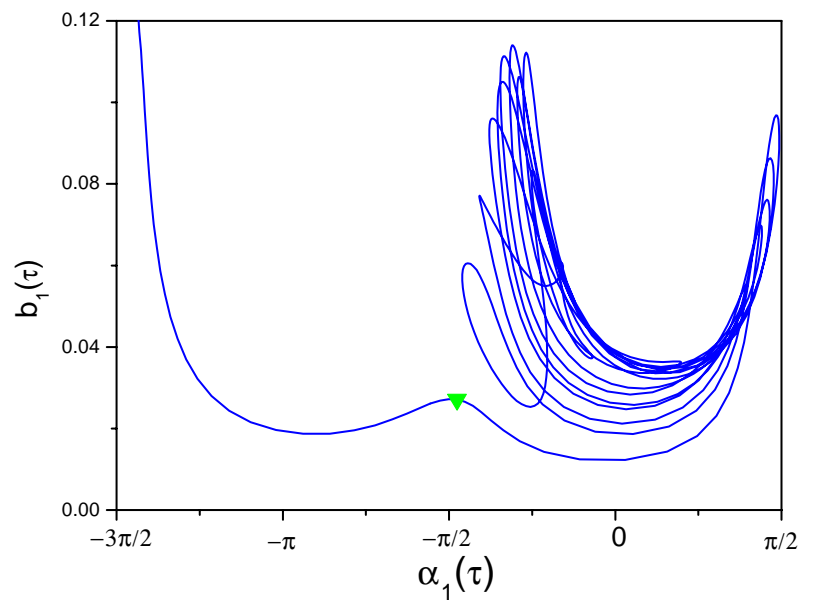

Fig. 6. Motion of the master mode $b_{1}(\tau)$ vs. $\alpha_{1}(\tau)$, the same transient period as in Fig. 5. The triangles in Figs. 5 and 6 correspond to the same moment.

$2 \pi$ as well. The latter fact can be seen more clearly in Fig. 7 for temporal evolutions of Fig. 7a $b_{1}(\tau)$ and Fig. 7b $\alpha_{1}(\tau)$. The arrows in Figs. 7a and b correspond to the critical times for the two dynamic events, i.e. the collision and the turning point, respectively. One can see in Fig. 7b that after the second event $\alpha_{1}(\tau)$ can make vibrating as well as whirling $\operatorname{motion}($ here $\bmod (2 \pi)$ has been taken), in contrast, before the second event $\alpha_{1}(\tau)$ vibrates within a small range. In the inset we plot $\alpha_{1}(\tau)$ without taking $\bmod (2 \pi)$, one can see that after the second event $\alpha_{1}(\tau)$ steps down continuously.

The above results convince us that there are two critical dynamic events involved in the crisis-induced transition to the spatiotemporal chaos: the first one is a collision with the saddle point which triggers the crisis, the subsequent one is a state transition of $k=1$ mode phase, that is, its motion changes from a purely nonlinear vibration to a combined motion of whirling and vibrating, it is the latter event that directly spoils the spatial coherence and leads to the spatiotemporal chaos. In the calculations with different initial conditions we always observed the similar turning point and hump as the ones in Figs. 5 and 6, respectively, in particular the tops of the humps correspond to the same value of $\alpha_{1}$, i.e. $\alpha_{1}^{*} \approx-1.54$ (with $3.9 \%$ relative error in 20 test runs), suggesting that at this place there might be a saddle with a separatrix dividing the vibration and vibration-whirling regimes of $\alpha_{1}$, probably the second event is caused by a collision with this saddle.

\section{Functional relations between the modes in spatially regular wave before transition}

In the last section we transformed nonlinear wave Eq. (1) into a set of coupled oscillators $\left\{b_{k}(\tau), \alpha_{k}(\tau)\right\}$ moving in a potential $\phi_{0}(\xi)$, this transformation has greatly simplified the analysis for the nonlinear dynamics of our spatially extended system. Variations of waveforms observed in the system can 

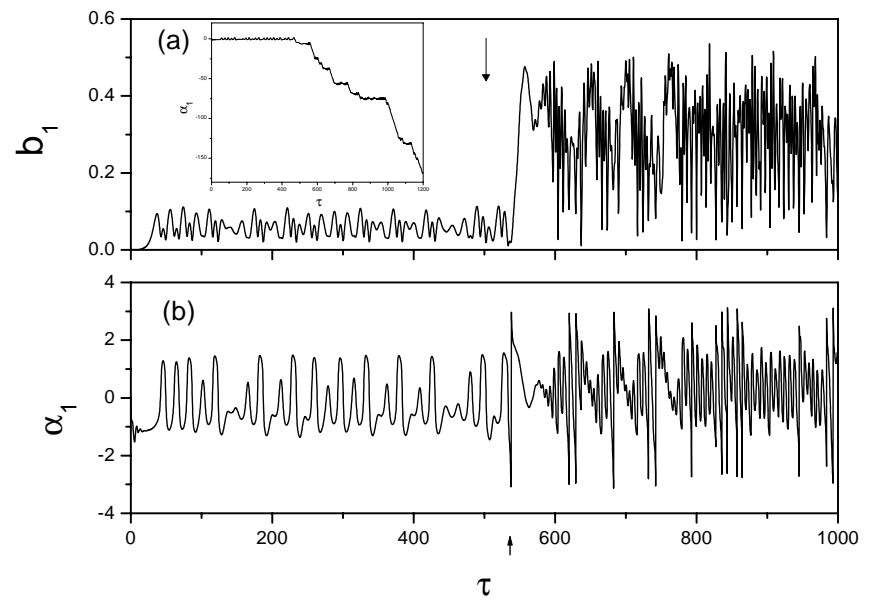

Fig. 7. Temporal evolution of (a) $b_{1}(\tau)$ and (b) $\alpha_{1}(\tau)$ for $\Omega=0.65, \varepsilon=0.22$, the two arrows indicate the critical times for the collision with the saddle point (the first critical event) and for the orbit ejected to the spatiotemporally chaotic attractor (the second critical event), respectively. The inset shows $\alpha_{1}(\tau)$ without taking $\bmod (2 \pi)$.

therefore be considered as the results of self-organization of the oscillators.

As is well known coupled nonlinear oscillators may adjust themselves to a PS under certain conditions, i.e. their phase difference $\left|\triangle \beta_{m n}(\tau)\right| \equiv\left|\beta_{m}(\tau)-\beta_{n}(\tau)\right|$ is constrained in a finite range (Fujisaka and Yamada, 1983; Pecora and Carroll, 1990; Rosenblum et al., 1996, 1997; Boccaletti et al., 2002). If asymptotically $\triangle \beta_{m n}$ is zero, the oscillators reach a complete PS; more generally $\beta_{m}(t)$ may show a functional PS with $\beta_{n}(t)$, i.e. while $\triangle \beta_{m n}(\tau)$ is small, a functional relation $\beta_{m}(t)=F\left[\beta_{n}(t)\right]$ is hold. In addition, a PS can be perfect or imperfect, for example, it is found that in coupled Lorenz oscillators, although the phase difference looks very erratic it can be confined in a finite range less than $2 \pi$ for a long time, and occasionally a phase slip may occur (Zaks et al., 1999).

Now let us compare the behaviors before and after the transition in our system in terms of PS. In the present section the spatially regular states for $\varepsilon<\varepsilon_{c}$ will be discussed. In our case the simplest spatial regular wave is a stable steady wave. From Eq. (5) with a saddle steady wave $\phi_{0}^{*}(\xi)$ as the potential, in certain parameter regimes we can find nontrivial constant solutions of $\left\{b_{k}(\tau \rightarrow \infty), \alpha_{k}(\tau \rightarrow \infty)\right\}$ corresponding to a stable steady wave $\phi_{0}(\xi)$, in which asymptotic phase difference $\Delta \alpha_{k k^{\prime}}(\tau \rightarrow \infty) \equiv \alpha_{k}(\tau)-\alpha_{k^{\prime}}(\tau)_{\mid \tau \rightarrow \infty}$ between any two oscillators is constant.

With variation of parameters $\Delta \alpha_{k k^{\prime}}(\tau)$ can no longer hold as constants, however, the oscillators are still able to adjust themselves to perfect functional PS. For $\Omega=0.65, \varepsilon=0.12$ Fig. 8 depicts Fig. 8a energy of the oscillators, $\delta E(\tau)=\sum_{k}\left(1-a k^{2}\right)\left[A_{k} b_{k} \cos \left(\theta_{k}-\alpha_{k}\right) / 2+b_{k}^{2} / 4\right]$, Figs. 8b-d phase difference $\Delta \alpha_{1 k}(\tau)$ for $k=2,3,4$, respectively. One can see that all the $\Delta \alpha_{1 k}(\tau)$ are confined within a finite range less than $2 \pi$. For the same parameters in Fig. 9 we plot $\alpha_{k}(\tau)$ vs. $\alpha_{1}(\tau)$ with Figs. 9a-c for $k=2,3,4$, respec-
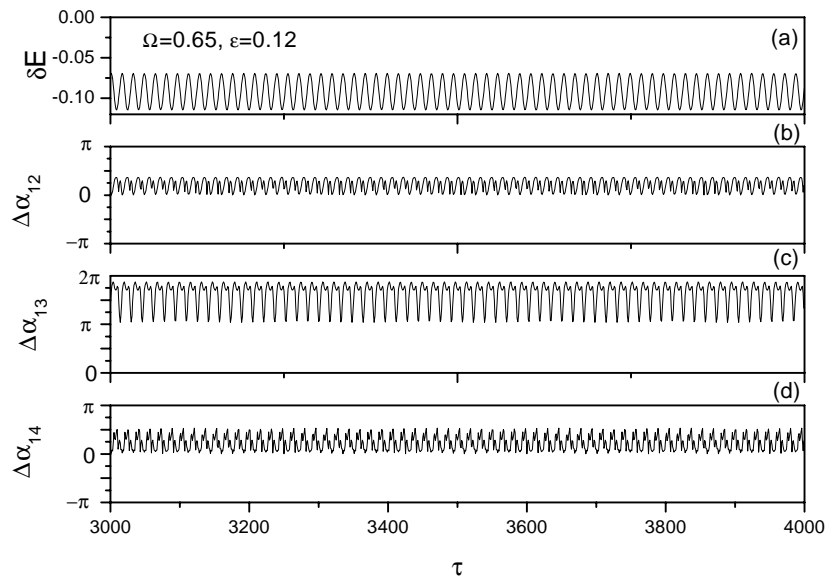

Fig. 8. Temporal evolution of (a) $\delta E(\tau)$, (b)-(d) $\Delta \alpha_{1 k}(\tau)$ for $k=2,3,4$, respectively, $\Omega=0.65, \varepsilon=0.12<\varepsilon_{c}$.

tively, they all show smooth curves, indicating that the oscillators have established a perfect functional PS collectively. As a result of the functional PS, $\delta E$ varies periodically as can be seen in Fig. 8a.

With increasing $\varepsilon$ the functional PS curves in the phase plot $\alpha_{k}(\tau)$ vs. $\alpha_{1}(\tau)$ become more and more complicated, and phase slips may occur in some oscillators. For $\Omega=0.65, \varepsilon=0.19$. Figure 10 shows $\alpha_{k}(\tau)$ with Fig. 10a $k=1$ (black), 2 (red), 4 (green) and Fig. 10b $k=1$ (black), 3 (pink). In the calculation $\bmod (2 \pi)$ is taken only when $\tau<3000$. One can see that in Fig. 10a, roughly speaking, both $k=2,4$ modes are synchronized with $k=1$ mode, occasionally $k=4$ mode experiences a phase slip but it is soon locked back to the domain of $\alpha_{1}(\tau)$ again; on the other hand, in Fig. 10b $\alpha_{3}(\tau)$ is no longer locked to the domain of $\alpha_{1}(\tau)$, which increases with time, in contrast to constant average of $\alpha_{1}(\tau)$. However, if taking $\bmod (2 \pi)$ for $\alpha_{3}(\tau)$ or $\alpha_{4}(\tau)$, respectively, one would find that functional relations of their motion with that of the other modes still remain. Figure 11 shows the phase plot $\alpha_{k}(\tau)$ vs. $\alpha_{1}(\tau)(k=2,3,4$ in Figs. 11a-c, respectively) for the same case as in Fig. 10 but with the $\bmod (2 \pi)$ taken, in all the plots we obtain smooth curves, that is, they have perfect functional relations. In the inset of Fig. 10b we show $\Delta \alpha_{13}(\tau) \equiv \alpha_{1}(\tau)-\alpha_{3}(\tau)$, one can see that the phase slip of $\alpha_{3}(\tau)$ relative to $\alpha_{1}(\tau)$ occurs nearly in every characteristic period, nevertheless the staircase-like behavior of $\triangle \alpha_{13}(\tau)$ suggests that $\alpha_{3}(\tau)$ also reaches a kind of functional PS with $\alpha_{1}(\tau)$.

As $\varepsilon$ increases further, temporal variation of the wave becomes more and more erratic, and the functional relations among $\alpha_{k}$ 's also become very complicated; when near the critical point $\varepsilon_{c}$, the functional relations may disappear intermittently, which induces a temporal loss of the spatial regularity. These phenomena are not in the scope of the present paper. Regardless of these problems, from the above results we can say that for producing a smooth waveform the mode phases are not necessary to be locked within a small range, with phase slips in some modes the oscillators in potential 

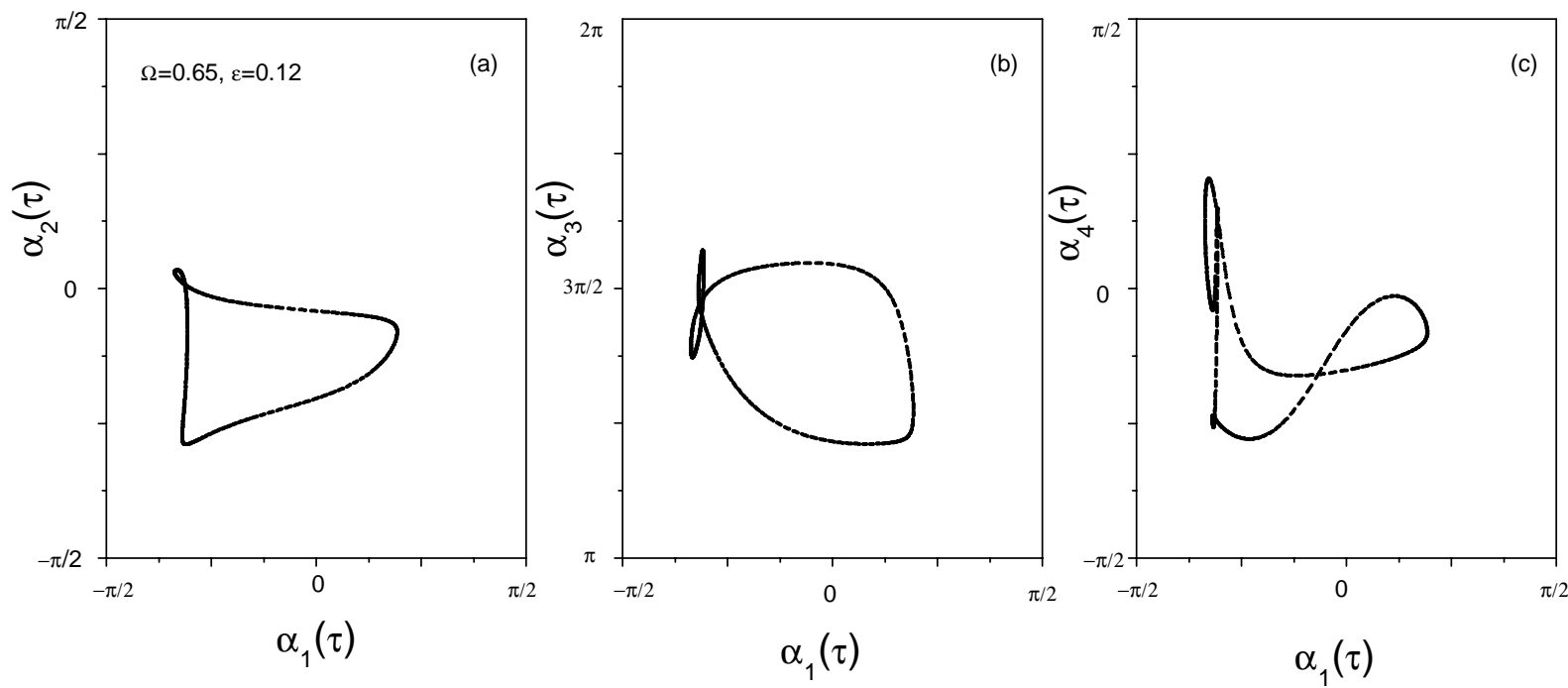

Fig. 9. $\alpha_{k}(\tau)$ vs. $\alpha_{1}(\tau)$ for the same case as in Fig. 8, (a)-(c) are for $k=2,3,4$, respectively.
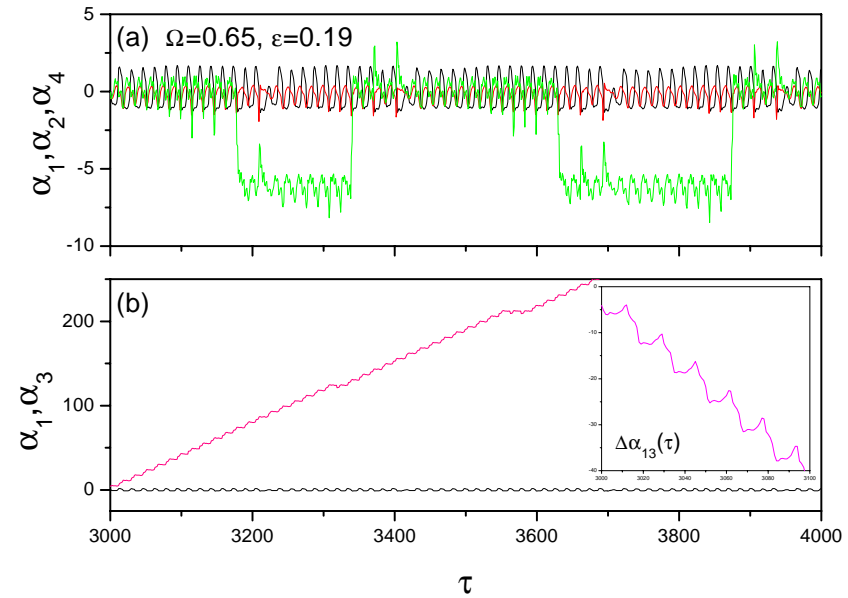

Fig. 10. Temporal evolution of $\alpha_{k}(\tau)$ for (a) $k=1$ (black), 2 (red), 4 (green), (b) $k=1$ (black), 3 (pink), $\Omega=0.65, \varepsilon=0.19<\varepsilon_{c}$. The inset shows $\Delta \alpha_{13}(\tau)$.

$\phi_{0}^{*}(\xi)$ can still adjust their relative relations in a perfect functional way, producing a wave that is erratic in time while maintaining the spatial coherence.

\section{On-off imperfect phase synchronization in spatiotem- poral chaos}

The wave state after the transition is extremely irregular both in time and in space. It is remarkable that even in such turbulent waves one can observe PS. However, now the synchronization is characterized by its imperfection (He and Chian, 2003). Just like in coupled Lorenz oscillators (Zaks et al., 1999), a turbulent wave in our system has an embedded saddle point, which has a characteristic time scale unbounded from above, the oscillators are not easy to adjust to a perfect PS.
Figure 12 is an example with $\Omega=0.65, \varepsilon=0.22>\varepsilon_{c}$, where Fig. 12a is the temporal evolution of $\delta E(\tau)$, Figs. 12b-d are phase differences $\Delta \alpha_{1 k}(\tau)$ for $k=2,3,4$, respectively. One can see that in $\delta E(\tau)$ there are many sharp spikes, corresponding to each sharp spike in Fig.12a, all the phase differences $\Delta \alpha_{1 k}$ in Figs. 12b-d are varying erratically in a very small range; in contrast, between two sharp spikes $\delta E(\tau)$ oscillates in a lower level and $\Delta \alpha_{1 k}$ can become very large, even surpassing $2 \pi$, (in Figs. $12 \mathrm{~b}-\mathrm{d} \bmod (2 \pi)$ has been taken). That is, in this turbulent wave the phases $\alpha_{k}(\tau)$ can adjust to imperfect PS intermittently. We name this behavior as an on-off collective imperfect PS (He and Chian, 2003).

The correspondence between the spikes in $\delta E(\tau)$ and imperfect PS in $\alpha_{k}(\tau)$ is significant, which suggests that bursts in the wave energy are induced by the imperfect PS "on". To further confirm this point, let us observe the motion of the mode amplitudes $\left\{b_{k}(\tau)\right\}$. An individual $b_{k}(\tau)$ looks very chaotic, however, if putting different mode amplitudes together one would find that synchronization may also exist among them. Figure 13 is for the same parameters as in Fig. 12, with Fig. 13a $\delta E(\tau)$, Fig. $13 \mathrm{~b} \alpha_{k}(\tau)$ for $k=1,2$ and Fig. 13c $b_{k}(\tau)$ for $k=1,2,3$, respectively. In the plot one can see that, while the phases $\left\{\alpha_{k}\right\}$ adjust to a synchronization the amplitudes $\left\{b_{k}\right\}$ reach maximum about simultaneously, due to effective building up of the mode energies, a burst appears in $\delta E(\tau)$, respectively in "on" stages of imperfect PS. This phenomenon is very different from that of spatially regular state before the transition. For a spatially regular wave, a PS among the oscillators is perfect (see Figs. 9 and 11); besides, due to the functional relation between them, normally the peaks of $\delta E(\tau)$ do not show correspondence to small $\Delta \alpha_{k k^{\prime}}(\tau)$, and $\left\{b_{k}(\tau)\right\}$ do not reach maximum simultaneously, therefore the mode energies can not be effectively added up. From this comparison we believe that such a turbulence with on-off collective imperfect PS is possibly a state where the total wave energy can be most effectively built 

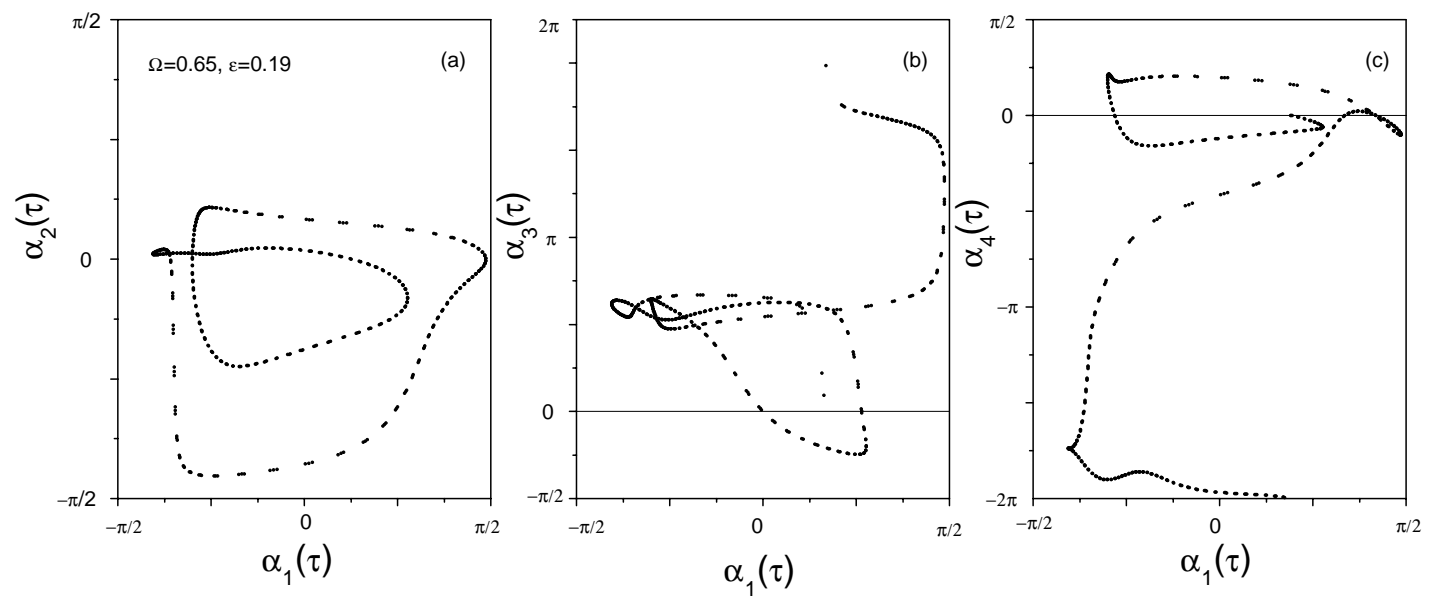

Fig. 11. $\alpha_{k}(\tau)$ vs. $\alpha_{1}(\tau)$ for the same case as in Fig. 10, (a)-(c) are for $k=2,3,4$, respectively, $\bmod (2 \pi)$ has been taken.

up by its mode energies of different scales, although only in short periods of time. In the "on" stages a best cooperation is realized between the oscillators.

In Fig. 14 we plot temporal variation of $\Delta \alpha_{23}(\tau)$ without taking $\bmod (2 \pi)$, some plateaus can be seen in the curve, which correspond to the "on" stages of collective imperfect PS; between two plateaus $\left|\triangle \alpha_{23}\right|$ increases very quickly, which are the "off" stages of the PS. Because of the multidimensions of our system, the plateaus are not as flat as in the coupled Lorenz oscillators (Zaks et al., 1999), and usually in our case the oscillators take a longer time to adjust to a new plateau again.

As we have seen in Sect. 2, both before and after the crisis the system has a saddle point, so the different PS behaviors in the spatially regular wave and spatiotemporal chaos can not be explained only by the existence of saddle point. Notice that owing to the second dynamic event the $k=1$ oscillator experiences a trapped-free transition, delocalization of this master mode is also crucial for these different behaviors. Before the transition the motion of $\left\{b_{k}, \alpha_{k}\right\}$ is strongly governed by the potential $\phi_{0}^{*}(\xi)$, a functional relation is then formed between the modes; after the transition, the $k=1$ mode which slaves the other modes - can be free from the potential, in the meantime the amplitudes $\left\{b_{k}\right\}$ grow greatly, in this case the potential has less influence and so the oscillators have a stronger tendency to adjust by themselves, as a result an on-off collective imperfect PS is established.

In the "on" stages of collective imperfect PS $\Delta \alpha_{k k^{\prime}}$ is near 0 or $\pi$, this fact allows us to define a correlation function between the phases of $N$ oscillators

$C_{\alpha}^{N}(\tau)=\prod_{k=1}^{N}\left|\cos \alpha_{k}(\tau)\right|$

Figure 15 shows (a) the wave energy $\delta E(\tau),(\mathrm{b})\left\langle C_{\alpha}^{4}(\tau)\right\rangle$, (c) $\left\langle C_{\alpha}^{5}(\tau)\right\rangle$, here \langle\rangle denotes time-average in a characteristic period. In the plot correspondence between the sharp spikes of $\delta E(\tau)$ and that of $\left\langle C_{\alpha}^{N}(\tau)\right\rangle$ is obvious, in particular in (b) $\left\langle C_{\alpha}^{4}(\tau)\right\rangle$ shows strong spikes whenever there appears a burst

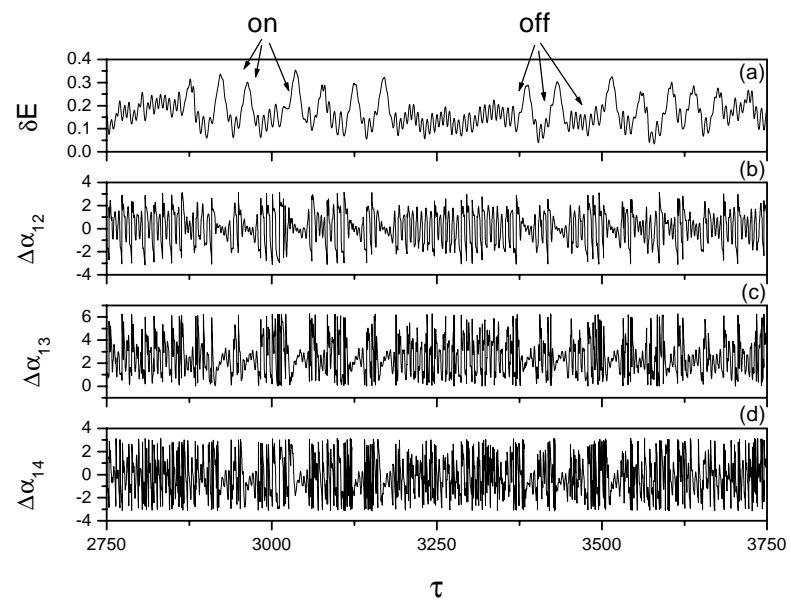

Fig. 12. Temporal evolution of (a) $\delta E(\tau)$, (b)-(d) $\Delta \alpha_{1 k}(\tau)$ for $k=2,3,4$, respectively, $\Omega=0.65, \varepsilon=0.22>\varepsilon_{c}$. One can see that whenever imperfect PS occurs a strong burst appears in $\delta E(\tau)$.

in $\delta E(\tau)$. In (c) the spikes of $\left\langle C_{\alpha}^{5}(\tau)\right\rangle$ are still apparent, but is not as prominent as in $\left\langle C_{\alpha}^{4}(\tau)\right\rangle$. If more and more modes are included the spikes in $\left\langle C_{\alpha}^{N}(\tau)\right\rangle$ is gradually smeared out. It is likely that the best synchronization occurs among a few long wavelength modes. Apart from those strong spikes there are also smaller peaks in $\left\langle C_{\alpha}^{N}(\tau)\right\rangle$, the correspondence between the peaks of $\left\langle C_{\alpha}^{N}(\tau)\right\rangle$ and that of $\delta E(\tau)$ is also clear. These results indicate that in the turbulent state after the crisis, an instant wave energy significantly depends on the relative phase status of the oscillators, in particular when the oscillators adjust to a collective imperfect PS the total energy displays a strong burst.

We expect that the statistics of $C_{\alpha}^{N}(\tau)$ can reflect that of $\delta E(\tau)$ to certain extent. Based on this consideration we calculate probability of interspikes of $C_{\alpha}^{N}(\tau)$. Figure 16 is an example of probability function $P\left(\tau_{L}\right)$ obtained from $C_{\alpha}^{4}(\tau)$, here interspikes $\tau_{L}$ are determined by a threshold 0.01 . One can find a very good power law $P \sim \tau_{L}^{-\alpha}$ as fitted by the straight line. 


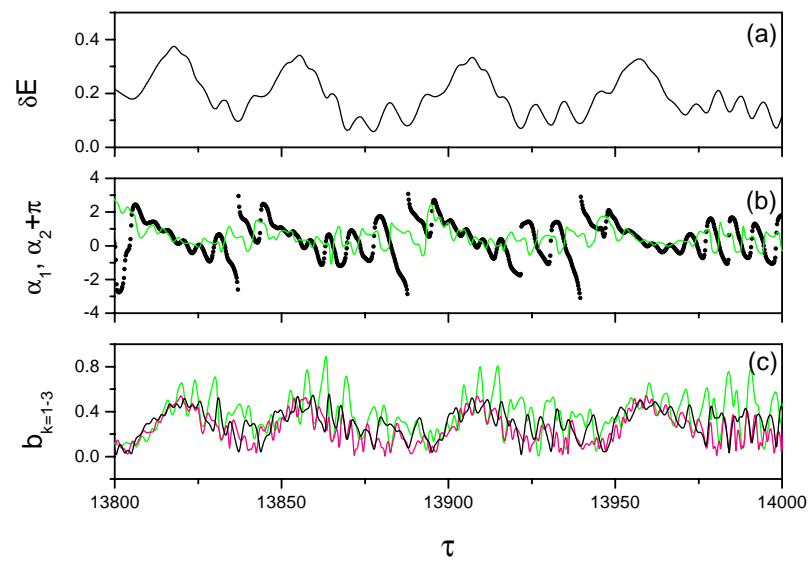

Fig. 13. Temporal evolutions of (a) $\delta E(\tau)$, (b) $\alpha_{k}(\tau)(k=1,2)$ and (c) $b_{k}(\tau)(k=1-3)$, for $\Omega=0.65, \varepsilon=0.22$, black/green/red line is for $k=1,2,3$, respectively.

\section{Conclusion and discussion}

To investigate nonlinear dynamics of the drift-wave system, we transform the nonlinear wave into a set of coupled oscillators in the potential of a steady wave solution. These oscillators contribute the active part of the wave. In particular the motion of oscillators in the potential of a saddle steady wave is studied, the latter is a saddle point in its moving frame. It is shown that a collision with the saddle point (or equivalently a "pattern resonance" with the embedded saddle steady wave) triggers a crisis, which then induces a subsequent event that destroys the spatial regularity of the wave.

The phase dynamics is investigated for the states before and after the crisis-induced transition. We find that in the very turbulent state after the transition the oscillators have a strong tendency of adjusting to PS, as a result an on-off imperfect PS can be established. As a comparison in the spatially regular states before the transition, perfect functional PS among the oscillators are observed with or without phase slips. The different behaviors of mode phases can be understood if taking into account the effect of the saddle steady wave potential $\phi_{0}^{*}(\xi)$, which strongly governs the motion of the oscillators before the transition but has less influence after it. In the latter case the master mode can be free of the potential, the orbits of oscillators are no longer restricted by a simple functional relation, instead they have more opportunities to adjust their amplitudes and phases, consequently a special on-off collective imperfect PS is formed.

The transformation of nonlinear wave Eq. (1) into Eq. (4) is mathematically strict, besides, when obtaining the coupled oscillators Eqs. (5) we did not make further assumption for the wave solutions except for the periodicity. The dynamic phenomena observed in Eq. (5) with appropriate truncation are in agreement with that of Eq. (1). One can therefore believe that the phase dynamics obtained in Eq. (5) reveals the physical process in nonlinear wave Eq. (1).

When dealing with turbulence, usually a random-phase as-

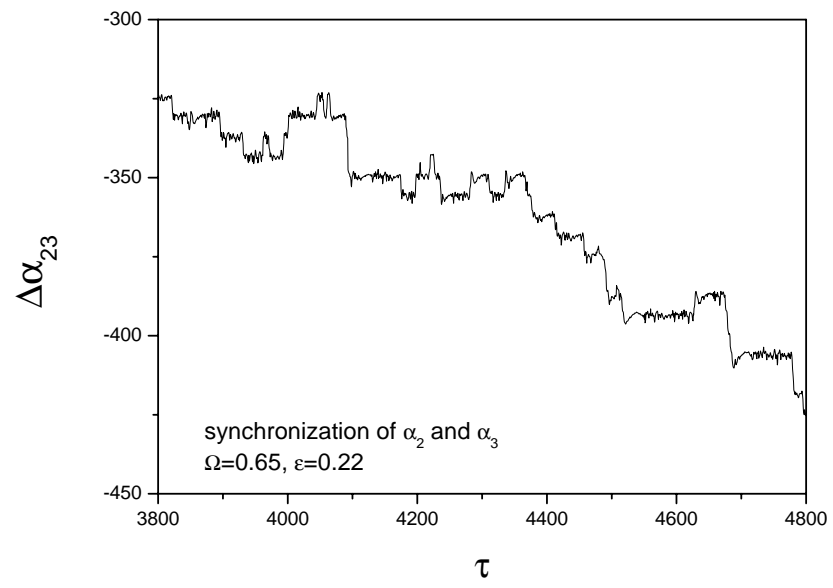

Fig. 14. Temporal evolution of $\Delta \alpha_{23}(\tau)$ for $\Omega=0.65, \varepsilon=0.22$ without taking $\bmod (2 \pi)$.

sumption is adopted in statistical investigations. However, our results indicate that the mode phases are not really random even in a very turbulent state. In our case phases $\left\{\alpha_{k}\right\}$ are of the oscillators, which are obtained after transforming to the moving frame and subtracting the saddle steady wave. Nevertheless, it is evident now that different spatial scales in a turbulence are not statistically uncorrelated, on the contrary, they may intermittently show very strong correlations both in their amplitudes and phases, the instant correlations can be even stronger than in spatially regular waves. That is why energy bursts show up in the turbulent waves rather than in spatially regular ones. In the real world similar energy bursts can be seen in many turbulent systems, e.g. swells in ocean, flares in the sun, sharp spikes in brain electric signals ..., can these bursts also be attributed to mode energy building up in "on" stages of the PS? If this is proved to be true, on-off collective imperfect PS may provide a potential application in predicting occurrence of energy bursts in these systems. For instance, solar flares are considered as a result of nonlinear solar turbulent plasmas, furthermore, they display the same statistics as that of $C_{\alpha}^{N}$, e.g. probability distribution of interspikes of the flares also follows a power law (Boffetta et al., 1999). If on-off collective imperfect PS is shown to be the mechanism of solar turbulence, in principle solar flares should be predictable. However, to this end we may need the knowledge of, e.g. the embedded saddle steady wave, with which one is able to transform the system to the moving frame and work out the motion of oscillators that constitute the active part of the turbulent wave. Obviously for practical application we still face many difficulties.

The study carried out in this paper has important application in geosciences. The earth-oceans-space system is dominated by turbulence where nonlinear processes govern the complex behaviors of the system (Chian and the WISER team, 2003; Chian et al., 2003b). The relevant investigations indicated that PS can occur in these systems. For example, Hada et al. used a method based on surrogate data technique and fractal analysis to evaluate the phase coherence 


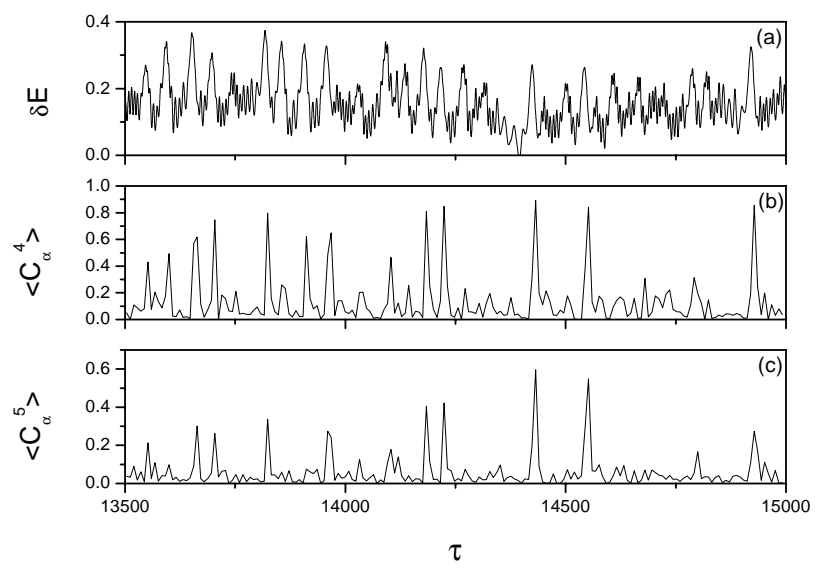

Fig. 15. Temporal evolution of (a) $\delta E(\tau)$ and (b), (c) $\left\langle C_{\alpha}^{N}(\tau)\right\rangle$ for $N=4,5$, respectively, $\Omega=0.65, \varepsilon=0.22$. The correspondence of the bursts in $\delta E(\tau)$ with spikes of $\left\langle C_{\alpha}^{4}(\tau)\right\rangle$ is very clear.

of magnetohydrodynamic turbulence in the earth's foreshock region, and showed that the correlation of wave phases exists which provides an evidence of nonlinear wave interactions (Hada et al., 2003). Koga and Hada applied the wavelet analysis to show that although the magnetohydrodynamic turbulence in the Earth's foreshock region is consisted of waves of a wide frequency range, only frequencies lower than the ion gyrofrequency are responsible for generating the phase coherence (Koga and Hada, 2003). Schulz et al. suggest that phase synchronization of different climate cycles can explain glacial-interglacial contrast in ocean climate variability (Schulz et al., 2004). The space observation of Hada et al. (2003) and Koga and Hada (2003) and the ocean model of Schulz et al. (2004) are in agreement with our theoretical results that in general PS plays an important role in nonlinear wave systems. In particular, our result on the on-off collective imperfect PS may help for further investigation on synchronization of different spatial scales in very turbulent systems.

Acknowledgements. This work is supported by the Special Funds for Major State Basic Research Projects of China and by the NSFC No. 10475009 and No. 10335010, by RFDP No. 20010027005 in China, and by CNPq in Brazil.

Edited by: P. Chu

Reviewed by: one referee

\section{References}

Benjamin, T. B., Bona, J. L., and Mahony, J. J.: Model equations for long waves in nonlinear dissipative systems, Philos. Trans. Roy. Soc. London Ser. A, 272, 47-78, 1972.

Bishop, A. R., Fesser, K., Lomdahl, P. S., Kerr, W. C., Williams, M. B., and Trullinger, S. E.: Coherent spatial structure versus time chaos in a perturbed Sine-Gordon system, Phys. Rev. Lett., 50, 1095-1098, 1983.

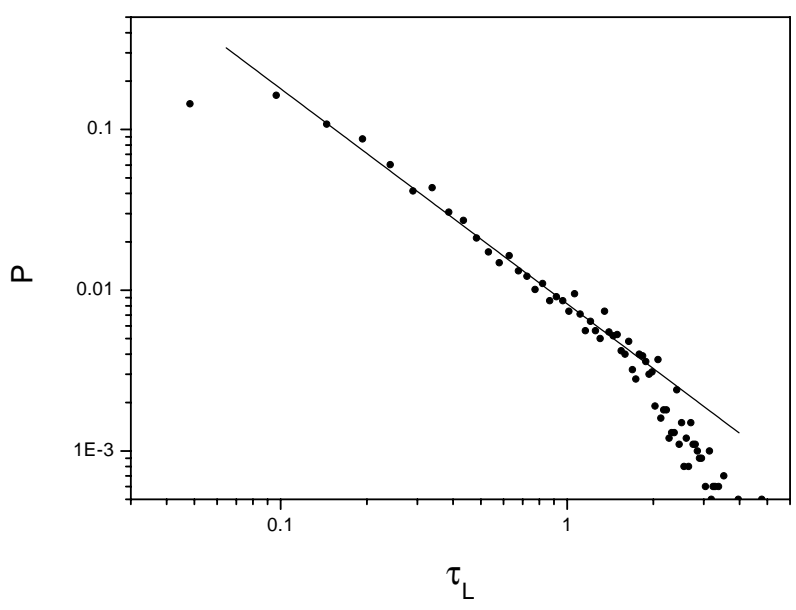

Fig. 16. Distribution function, $P\left(\tau_{L}\right)$, of interspike $\tau_{L}$ of $\left\langle C_{\alpha}^{4}(\tau)\right\rangle$ for $\Omega=0.65, \varepsilon=0.22$, which shows a power law behavior as fitted by the solid line.

Biskamp, D. and He, K.: Three-drift-wave interaction at finite parallel wavelength: bifurcation and transition to chaos, Phys. Fluids, 28, 2172-2180, 1985.

Boccaletti, S., Kurths, J., Osipov, G., Valladares, D. L., and Zhou, C. S.: The synchronization of chaotic systems, Phys. Rep., 366, 1-101, 2002.

Boffetta, G., Carbone, V., Giuliani, P., Veltri, P., and Vulpiani, A.: Power laws in solar flares: Self-organized criticality or turbulence?, Phys. Rev. Lett., 83, 4662-4665, 1999.

Chaté, H. and Manneville, P.: Transition to turbulence via spatiotemporal intermittency, Phys. Rev. Lett., 58, 112-115, 1987.

Chern, C. and I, L.: Multiplicity of bifurcation in weakly ionized magnetoplasmas, Phys. Rev. A, 43, 1994-1997, 1991.

Chian, A. C.-L., Rempel, E. L., Macau, E. E., Rosa, R. R., and Cristiansen, F.: High-dimensional interior crisis in the KuramotoSivashinsky equation, Phys. Rev. E, 65, 035203(R)-1-4, 2002.

Chian, A. C.-L. and the WISER team: Foreword: advances in space environment research, Space Science Rev., 107, 1-3, 2003.

Chian, A. C.-L., Borotto, F. A., Rempel, E. L., Macau, E. E. N., Rosa, R. R., and Christiansen, F.: Dynamical systems approach to space environment turbulence, Space Science Rev., 107, 447461, 2003.

Cross, M. C. and Hohenberg, P. C.: Pattern formation outside of equilibrium, Rev. Mod. Phys., 65, 851-1112, 1993.

Dodd, R. K., Eilbeck, J. C., Gobbon, J. D., and Morris, H. C.: Solitons and nonlinear wave equations, Academic Press, London, 596, 1982.

Eckmann, J. -P.: Roads to turbulence in dissipative dynamical systems, Rev. of Mod. Phys., 53, 643-654, 1981.

Foss, J., Longtin, A., Mensour, B., and Milton, J. : Multistability and delayed recurrent loops, Phys. Rev. Lett., 76, 708-711, 1996.

Frisch, U.: Turbulence, Cambridge University Press, Cambridge, 1995.

Fujisaka, H. and Yamada, T.: Stability theory of synchronized motion in coupled-oscillator systems, Prog. Theor. Phys., 69, 32-47, 1983.

Hada, T., Koga, D., and Yamanoto, E.: Phase coherence of MHD waves in the solar wind, Space Science Rev., 107, 463-466, 2003.

He, K. and Salat, A.: Hysteresis in the nonlinear driven drift and 
Korteweg-de Vries equation, Phys. Lett. A, 132, 175-178, 1988. He, K. and Salat, A.: Hysteresis and onset of chaos in periodically driven nonlinear drift waves, Plasma Phys. Contr. Fusion, 31, 123-141, 1989.

He, K.: Crisis-induced transition to spatiotemporally chaotic motions. Phys. Rev. Lett. 80, 696-699, 1998.

He, K.: Saddle pattern resonance and onset of crisis to spatiotemporal chaos, Phys. Rev. Lett., 84, 3290-3293, 2000.

He, K. and Chian, A. C. -L.: On-off collective imperfect phase synchronization and bursts in wave energy in a turbulent state, Phys. Rev. Lett., 91, 034102-1-4, 2003.

He, K.: Hopf bifurcation in a nonlinear wave system, Chin. Phys. Lett., 21, 439-442, 2004.

He, K. and Chian, A. C. -L.: Critical dynamic events at the crisis of transition to spatiotemporal chaos, Phys. Rev. E, 69, 026207-112, 2004.

Horton, W.: Nonlinear drift waves and transport in magnetized plasmas, Phys. Rep., 192, 1-177, 1990.

Infeld, E. and Rowlands, G.: Nonlinear waves, solitons and chaos, Cambridge University Press, Cambridge, 1990.

see, for example, Principles of Neural Science, edited by Kandel, E. R., Schwartz, J. H. and Jessell, T. M., The Mc Graw-Hill Companies, Inc., 938, 2000.

Kim, S., Park, S. H., and Ryu, C. S.: Multistability in coupled oscillator systems with time delay, Phys. Rev. Lett., 79, 2911-2914, 1997.

Klinger, T., Latten, A., Piel, A., Bonhomme, G., Pierre, T., and Dudok de Wit, T.: Route to drift wave chaos and turbulence in a bounded low- $\beta$ plasma experiment, Phys. Rev. Lett., 79, 39133916, 1997.

Koga, D. and Hada, T.: Phase coherence of foreshock MHD waves: wavelet analysis, Space Science Rev., 107, 495-498, 2003.
Lichtenberg, A. J. and Lieberman, M. A.: Regular and Chaotic dynamics, Springer, Berlin), 1983.

Nakatsuka, H., Asaka, S., Itoh, H., Ikeda, K., and Matsuoka, M.: Observation of bifurcation to chaos in an all-optical bistable system, Phys. Rev. Lett., 50, 109-112, 1983.

Ott, E.: Chaos in dynamical systems, Cambridge University Press, New York, 277, 1993.

Park, E.-H., Zaks, M. A., and Kurths, J.: Phase synchronization in the forced Lorenz system, Phys. Rev. E, 60, 6627-6638, 1999.

Pecora, L. M. and Carroll, T. L.: Synchronization in chaotic systems, Phys. Rev. Lett., 64, 821-824, 1990.

Rosenblum, M. G., Pikovsky, A. S., and Kurths, J.: Phase synchronization of chaotic oscillators, Phys. Rev. Lett., 76, 1804-1807, 1996.

Rosenblum, M. G., Pikovsky, A. S., and Kurths, J.: From phase to lag synchronization in coupled chaotic oscillators, Phys. Rev. Lett., 78, 4193-4196, 1997.

Schulz, M., Paul, A., and Timmermann, A.: Galcialinterglacial contrast in climate variability at centennial-tomilennial timescales: observations and conceptual model, Quaternary Science Rev., 23, 2219-2230, 2004.

Sun, H., Ma, L., and Wang, L. : Multistability as an indication of chaos in a discharge plasma, Phys. Rev. E, 51, 3475-3479, 1995.

Swinney, H. L.: Observation of order and chaos in nonlinear systems, Physica D, 7, 3-15, 1983.

Zaks, M. A., Park, E. -H., Rosenblum, M. G., and Kurths, J.: Alternating locking ratios in imperfect phase synchronization, Phys. Rev. Lett., 82, 4228-4231, 1999.

Zhou, L. and He, K.: Multistable structure in Korteweg-de Vries system driven by a solitary wave, Phys. Lett. A, 241, 159-162, 1998. 